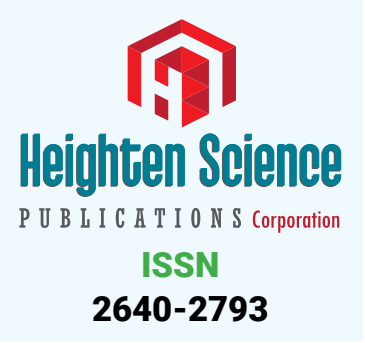

*Address for Correspondence: Nina Ivanovska Institute of Microbiology, Department of Immunology, Bulgarian Academy of Science, 1113 Sofia, Bulgaria, Tel: (+359 2) 979 3195; Email: nina@microbio.bas.bg;

nivanovska@abv.bg

Submitted: 24 December 2018

Approved: 28 December 2018

Published: 31 December 2018

Copyright: () 2018 Ganova P, et al. This is an open access article distributed under the Creative Commons Attribution License, which permits unrestricted use, distribution, and reproduction in any medium, provided the original work is properly cited

Keywords: Zymosan-induced arthritis; Dendritic cells; Mesnchymal cells; Fibroblasts; Megakaryocytes

Check for updates
Research Article

\section{Differentiation of bone marrow cells in arthritic mice with decreased complement activity}

\author{
Petya Ganova and Nina Ivanovska* \\ Institute of Microbiology, Department of Immunology, Bulgarian Academy of Science, 1113 \\ Sofia, Bulgaria
}

\section{Abstract}

There is evidence that complement components induce cell migration in mesenchymal stem cells and regulate cytokine production in osteoblastic cells thus playing a regulatory role in normal bone formation. The aim of the present study was to investigate the involvement of complement system in the differentiation of bone marrow cells in complement-depleted model of rheumatoid arthritis (RA). Arthritis was induced by intraarticular injection of zymosan in cobra venom factor (CVF)-treated mice depleted of functional complement. The expression of different markers by bone marrow [1], on fibroblasts (CD29), mesenchymal cells (CD105), dendritic cells (CD14, CD86), osteoclasts (CD265), cells expressing Dectin1 (CD369) and megakaryocytes (CD62P) was determined by flowcytometry. The lack of functional complement activity at the point of arthritis initiation (day 3 ) lead to an increase of fibroblast and megakaryocyte populations, to a decrease of mature and dectin1 positive populations, while the number of mesenchymal cells was not changed, all compared to arthritic mice. Immunohistochemical staining showed that low complement activity diminished arthritis-induced generation of megakaryocytes and platelets in BM. Chronic inflammation during erosive conditions such as rheumatoid arthritis, leads to dysregulated differentiation and proliferation of bone cells, inflammation of synovial membrane and bone marrow, and degradation of cartilage and bone. Present results point that the lack of functional complement changed the ratio between different cell populations that can be used for determining the development and stage of rheumatoid arthritis and can help finding of new therapeutic approaches.

\section{Introduction}

Complement system is an essential part of the innate immunity for detection and elimination of microbes. Complement activation helps to fight infections or to degrade dead or damaged cells at sites of injuries and invasion of pathogens. In contrast, during chronic inflammatory conditions such as autoimmune diseases, excessive or persisting complement activation can help destructive processes [2,3]. Complement is involved in the development of acute and chronic phases of RA. Deposits of C3c and C9 components and elevated levels of mRNA for C3, factor B, C3aR, and C5aR were found in the synovium and in the intercellular matrix, simultaneously with a decreased expression of CD59, an inhibitor of membrane attack complex (MAC) formation $[4,5]$. The complement system plays an important role both in immune system and in bone development and homeostasis, and specifically influences osteoblast and osteoclast (OC) activity [6]. Complement activation might increase bone resorption via enhanced osteoclastogenesis, while recruitment of osteoblast precursors might support bone formation. Anti-C3 antibodies inhibited osteoclastogenesis when added at early time of culture, inhibiting the proliferation of precursor cells and early differentiation [7].

Mesenchymal stem cells are capable of self-replication and differentiation that can contribute to the regeneration of mesenchymal tissues, such as bone and cartilage [8]. 
MSCs are promising candidate for various tissue engineering applications to treat mild and moderate $\mathrm{OA}$ [9], as superior results have been achieved with greater quantity of MSCs injected [10]. They express a number of cell surface markers, including CD105 (endoglin) which is a membrane glycoprotein and a part of the transforming growth factor-receptor complex [11]. Its expression on BM stem cells ensures superior proliferation and differentiation of chondrocytes. Dendritic cells (DCs) are able to trigger MHC-restricted autoimmune responses in lymphoid organs as well as inflammation-associated DC maturation and activation may initiate T-cell cytokine production and B-cell antibody synthesis $[12,13]$. Plasmacytoid DCs are recruited to normal lymphoid organs as well as inflammatory sites, including RA synovial tissue where they undergo local differentiation. Myeloid DC precursors populate normal resting synovial tissues which is additionally enhanced in response to inflammatory chemokines. Dendritic-cell-associated C-type lectin-1 (Dectin-1) is a $\beta$-glucan receptor belonging to Dectin-1 family expressed on monocytes, macrophages, neutrophils and weakly on T cells, and B cells. Zymosan, a beta-glucan and mannan-rich ligand is one of Dectin-1 ligands that activates Dectin-1-SYK and Toll like receptor (TLR)2MyD88 signaling pathways [14]. Dectin-1 induces DC maturation and the production of cytokines, such as IL-23 independently of the TLRs [15]. Dectin-1 and MyD88coupled TLRs result in an enhanced production of IL-23, while the production of IL-12 is downregulated [16]. MKs develop from hematopoietic stem cells that reside mainly in the bone marrow. They account only for $0.01 \%$ of nucleated bone marrow cells [17]. MKs undergo a maturation process for five days in humans and for 2-3 days in rodents. They increase in size, become full of platelet-specific granules, increase their cytoplasmic content of cytoskeletal proteins. Binding of MKs to collagen I through $\alpha 2 \beta 1$-integrin inhibits proplatelet formation [18]. Platelets express a number of complement receptors, such as $\mathrm{C} 1 \mathrm{qR}, \mathrm{C} 3 \mathrm{aR}$, and $\mathrm{C} 5 \mathrm{aR}$ as well as $\mathrm{P}$ selectin via which they bind plasma complement proteins, whereby they become activated. It should be noted that platelets contain a specific form of C3 [19] and also express complement regulatory molecules such as CD59, factor $\mathrm{H}$, and decay acceleration factor, which prevent excessive complement activation on the platelet surface. Bone erosion is a result of the differentiation of mononuclear precursor cells to mature osteoclasts formed in the presence of macrophage-colony stimulating factor (M-CSF), and receptor activator of nuclear factor-kappaB ligand (RANKL) within the synovial membrane [20]. In the bloodstream osteoclast precursors under the action of certain chemokines released at sites of resorption settle into bones and differentiate into OCs [21]. They attach to bone surfaces by integrin vitronectin receptor $\alpha \mathrm{V} \beta 3$. When occupied, $\alpha_{v} \beta_{3}$ activates a canonical signaling complex consisting of c-Src, Syk, Dap12, Slp76, Vav 3, and Rac that permits the cell to spread and form actin rings. Absence of any component of this signaling pathway compromises osteoclast cytoskeletal organization and limits bone resorption [22].

Previously, using a model of complement depletion we showed that the absence of functional complement activity strongly inhibited the development of synovitis through decreased levels of C5a and sRANKL in the synovial fluid [23]. Also, we demonstrated that the depletion of functional complement activity at acute phase of arthritis significantly reduced the expression of TGF-beta1/3, BMP2 and pSmad2, and the excessive synthesis of proteoglycans and glycosaminoglycans [24]. The aim of the present study was to investigate the influence of complement activity on BM cell differentiation at the point of ZIA initiation.

\section{Materials and Methods}

\section{Animals}

Male and female Balb/c (8-10 week old, weight 20-22 g) were bred in the Animal Facility of the Institute of Microbiology, maintained on a 12:12 h light: dark cycle and fed standard diet and tap water ad libitum. In the experiments were used totally 60 
mice (each group consisted of 10 mice). All experiments were in accordance with the Bulgarian Agency Guidelines №352 06.01.2012 and approved by the Animal Care Committee at the Institute of Microbiology, Sofia.

\section{Zymosan-Induced Arthritis (ZIA)}

Zymosan A from Saccharomyces cerevisiae (Sigma, St Louis, MO, USA) was resuspended in distilled water $(40 \mathrm{mg} / \mathrm{ml})$ and the suspension was autoclaved and stored in aliquots at $-20^{\circ} \mathrm{C}$. Arthritis was induced by intra-articular (i.a.) injection of $180 \mu \mathrm{g}$ $(10 \mu \mathrm{l})$ of zymosan into the joint cavity under brief anaesthesia (sodium pentobarbital $50 \mathrm{mg} / \mathrm{kg}$, i.p.). Control animals received an i.a. injection of an equal volume of sterile phosphate buffered saline (PBS). After an initial peak of inflammation at about day 3 , it retarded by day 7 . Histologically, the joint inflammation was characterized by mononuclear cell infiltration in the sublining layer, cartilage damage as well as bone erosion [23].

For decomplementation mice were injected i.p. with $1 \mathrm{ng} / 20 \mathrm{~g}$ body weight of cobra venom factor (CVF, Sigma-Aldrich, St. Louis, MO) 72 and $48 \mathrm{~h}$ before the induction of zymosan arthritis. This scheme has been chosen according to our previous investigations where different concentrations of CVF and different repetitions were tested [23]. We achieved the lowest hemolytic complement activity under this scheme without mortality and 5 days were needed for restoration of normal levels of alternative pathway (AP) activity.

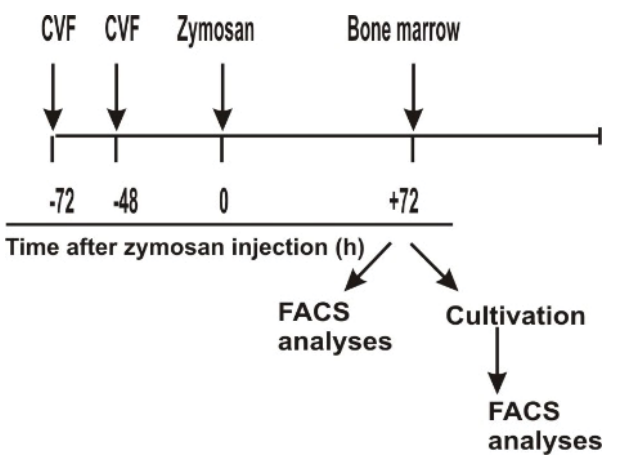

Design of the experiment

\section{Immunohistochemistry}

Mice were killed at day 3 and knees were dissected and fixed for 2 days in $4 \%$ buffered formalin. Fixed tissues were decalcified for 2 weeks in 15\% EDTA, dehydrated and embedded in paraffin. Sagittal sections $(5 \mu \mathrm{m})$ of the whole knee joint were stained with anti-mouse CD62P antibody (P-selectin, clone RMP-1, Biolegend). Further, slides were washed with PBS, blocked with 1\% BSA, washed and HRP-streptavidin 1:100 diluted; Sigma-Aldrich, Germany) was added for $10 \mathrm{~min}$. The slides were washed and incubated with DAB solution kit (3',3'diaminobenzididne kit, Abcam) for 10 minutes and counterstained with Gill's hematoxylin for 3 minutes.

\section{BM cell differentiation}

Femur and tibia of hind legs from adult mice were extracted, using a $27 \mathrm{G}$ needle/1 $\mathrm{ml}$ syringe filled with $\alpha$-minimal essential medium ( $\alpha$-MEM, Sigma Aldrich, Germany). The suspension was gently aspirated to disrupt cell aggregates and centrifuged at $1800 \mathrm{x}$ g for $5 \mathrm{~min}$. Part of the cells was analyzed for the expression of CD29, CD105, CD14, CD86, CD265 and CD369 markers.

Second part of BM precursors $\left(2 \times 10^{6} / \mathrm{ml}\right)$ were cultivated in $\alpha$-MEM supplemented with $10 \%$ FCS (Sigma-Aldrich, Germany), $5 \mathrm{mM}$ glutamine, $2.5 \mathrm{mM}$ HEPES, antibiotics (streptomycin $2 \mathrm{U} / \mathrm{ml}$, penicillin G $100 \mu \mathrm{g} / \mathrm{ml}$, amphotericine B $1 \mu \mathrm{g} / \mathrm{ml}$ (Sigma-Aldrich, Germany) for 1 day. Non-adherent cells were separated and adherent cells were 
cultivated in supplemented $\alpha$-MEM for 10 days with partial change of medium every $3^{\text {rd }}$ day. At the end of cultivation cells were collected and analyzed for the expression of CD29 and CD105 markers. Non-adherent cells were resuspended, and plated into 24-well culture plates at $2 \times 10^{6} / \mathrm{ml}$ cells $/ \mathrm{ml}$ and stimulated with recombinant mouse granulocyte-macrophage colony-stimulating factor (GM-CSF) (Biolegend, USA) at 10 $\mathrm{ng} / \mathrm{ml}$ plus recombinant IL- 4 at $0.3 \mathrm{ng} / \mathrm{ml}$ (Biolegend) and analyzed for the expression of CD14 and CD86 markers.

\section{Flow cytometry}

BM cells isolated as described above were stained with FITC anti-mouse CD29 (mesenhymal cells, clone HM( 1-1, Biolegend, USA), PE anti-mouse CD105 (fibroblasts, clone MJ7/18, Biolegend, USA), FITC anti-mouse CD14 (immature DCs, clone 5a 2-8, eBioscience), PE anti-mouse CD86 (mature DCs, clone B7-2, eBioscience), PE antimouse CD265 (RANK, clone R12-31, In vitro gen), PE anti-mouse CD62P (P-selectin, megakaryocytes, clone RPM-1, Biolegend, USA), APC anti-mouse CD369 (Dectin1, clone 7H1, Biolegend, USA). Nonspecific fluorescence was assessed by incubation with irrelevant isotype-matched conjugated mAbs. Flow cytometric analyses were performed by BD FACSDiva v6.1.2 Software (Becton Dickinson, NJ, USA) as follows. A gate was drawn around a live population of interest to isolate events for analysis. Data for events within this gate were then displayed in subsequent plots to determine the percentages of various subpopulations. A histogram allowed to view a single parameter against the number of events. Marker was placed above to designate positive events. A dot plot provided a two-parameter display of data. An isotype control was used to determine where the quadrant markers would be placed. A quadrant marker divided two-parameter plots into four sections to distinguish populations that were considered negative, single positive, or double positive. The upper-left quadrant contained events that were positive for the $y$-axis parameter (PE) but negative for the $x$-axis (FITC) parameter. The lower-right quadrant contained events that were positive for the $\mathrm{x}$-axis parameter (FITC) but negative for the $y$-axis (PE) parameter. The upper-right quadrant contained events that were positive for both parameters (double positive FITC+/PE+). The lower-left quadrant dysplayed events that were negative for both parameters.

\section{Statistics}

Statistical analysis was performed using InStat3.0 and GraphicPad Prism 5.0 (GraphPad Software Inc., La Jolla, CA, USA). Data were presented as means \pm SEM and comparisons between different groups were done using one-way analysis of variance (one way ANOVA). Values of $P<0.05$ were considered as statistically significant.

\section{Results}

In the present experiments BM cells obtained at day 3 of ZIA from treated or untreated with CVF mice were subjected to FACS analysis. We investigated the influence of complement depletion on fibroblast and mesenchymal cell differentiation. CD29 marker, characteristic for fibroblast-like cells was detected in higher degree in non-arthritic group, compared to arthritic mice. CVF-pretreatment resulted in elevated percentage of these cells, compared to arthritic but significantly lower, compared to nonarthritic (Figure 1A,C). Mesenchymal CD105 positive cells presented approximately 15 $\%$ in non-arthritic mice, while in arthritic and CVF-pretreated groups the percentage of positive cells presented $\sim 5 \%$ and $\sim 10 \%$, respectively (Figure 1B,D). Next, cells from the same groups were cultured for 10 days with partial change of supplemented media. FACS analysis showed that the differentiation of CD29+ fibroblast-like cells was inhibited in arthritic mice, less exerted in CVF-pretreated group (Figure 2A,C). Mesenchymal cell differentiation was also decreased in arthritic and CVF-treated groups (Figure 2B,D).

Also the differentiation of bone marrow DCs was determined in vivo and in vitro. 
Data showed that the percentage of immature CD14+ dendritic cells was elevated in non-arthritic mice compared to both, arthritic and CVF-treated mice. This inhibition was more expressed in CVF-group vs ZIA group (Figure 3A,B). Mature CD86+ cells presented in low percentages however decreased in ZIA group (Figure 3A,C). The percentage of double positive CD14+/CD86+ was higher in non-arthritic group compared to ZIA and CVF-treated groups (Figure 3A,D). Further, we investigated the influence of CVF-treatment on GM-CSF+IL-4 induced DC differentiation of BM cells in vitro. Results showed that the maturation of DCs is inhibited in the group of arthritic mice as evident from the increased percentage of CD14+ cells, compared to nonarthritic group (Figure 4A,B). In contrast, the percentage of mature CD86+ cells was very low in arthritic group, compared to non-arthritic once, while CVF-treated group had intermediate position. The pool of mature cells was higher compared to ZIA mice but lower, compared to non-arthritic mice (Figure 4A,C). Low percentage of double positive CD14+/CD86+ cells was found in non-arthritic group, significantly increased in ZIA group (Figure 4A,D).

In the present study we looked for the presence of RANK (CD265) positive cells in $\mathrm{BM}$. The results showed that in arthritic mice the percentage of RANK+ cells was higher than in non-arthritic mice, while CVF administration abolished this effect (Figure 5). The development of ZIA provoked an increase of CD62P+ cells that was augmented in CVF-pretreated group (Figure 6A,B). We also observed in non-arthritic mice a pool of Dectin1+ (CD369) cells. In zymosan-injected arthritic mice the percentage of CD369+ cells was elevated and the opposite effect was found in CVF-treated groups (Figure 6A,C). This result might be due to a rapid elimination of zymosan particles in decomplemented mice. MKs expressing Dectin1 (CD62P+/CD369+) were in very low amount in non-arthritic group while double positive cells were increased in arthritic group. This effect was weakly expressed in CVF-treated mice (Figure 6A,D). To elucidate the role of functional complement in the megakaryocyte differentiation we determined immunohistochemically the presence of CD62P+ cells in BM at day 3 of ZIA. In arthritic mice a massive influx of inflammatory cells was established together with giant megakaryocytes and groups of small CD62P+ platelets characteristic for this group while in CVF-treated mice there were only rare single giant cells (Figure 7).

\section{Discussion}

The pathophysiology of rheumatoid arthritis involves numerous different cell types, all of which contribute to the erosive processes. Activated synovial fibroblasts persist in large numbers in inflammed synovium being one of the key players in RA. They contribute to the progression of the disease through attaching to cartilage, invading into and leading to degradation of cartilage and bone $[25,26]$. In the rheumatoid synovium mRNA for all components of the alternative pathway (AP), except for properdin was detected. However, cultured synovial macrophages release C3 and properdin while fibroblast-like synoviocytes secreted factor B $[27,28]$. It is supposed that arthritis spreading is mediated in part, by the migration of activated synovial fibroblasts [29]. One possibility is BM cells to be pushed to mesenchymal or fibroblast differentiation already in BM supported by our observation that both populations were decreased in ZIA mice in freshly isolated BM suspension. Another possibility is the end differentiation to happen in tissues under appropriate mediators. This hypothesis was tested in vitro. BMs were cultivated for 10 days with partial change of medium in order to keep released mediators and we found that cells from ZIA mice were with decreased capacity to differentiate into mesenchymal or fibolast-like cells. This comparison supposed that most probably the differentiation in bone marrow and locally in tissues goes simultaneously.

DCs are likely to contribute in several ways to the pathogenesis of RA. They settle in the synovial tissue and synovial fluid where they present antigen locally, contributing to 

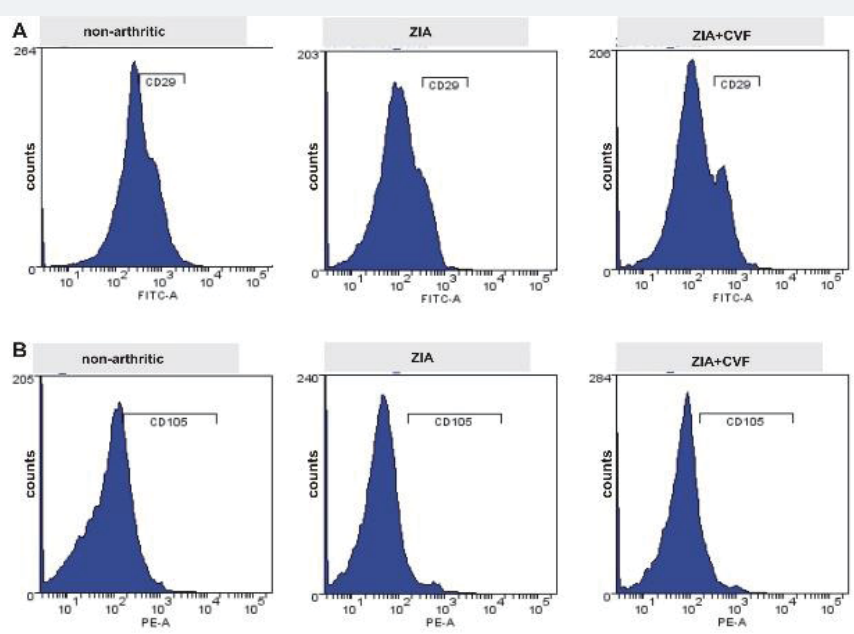

C

D
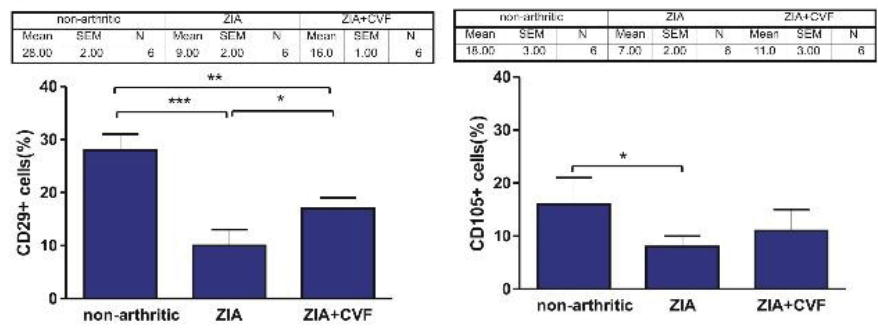

Figure 1: Effect of decomplementation on CD29 and CD105 expression. BM cells obtained from nonarthritic mice, mice with ZIA and pretreated with CVF mice with ZIA were collected at day 3 and the percentage of CD29+ cells (A) and CD105+ cells (B) was determined. Graphical presentation of CD29+ cells (C) and CD105 cells (D). Results are from two independent experiments (in each $n=10$ /per group) and expressed as means $\pm \mathrm{SEM}, * P<0.05,{ }^{\star} * P<0.01$, $\star \star \star P<0.001$, one way ANOVA.
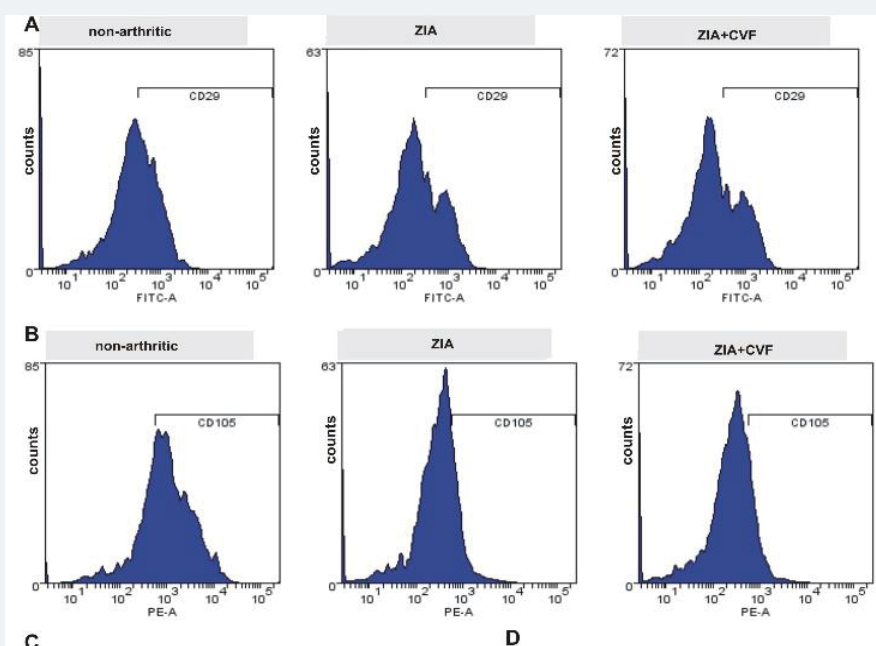

c
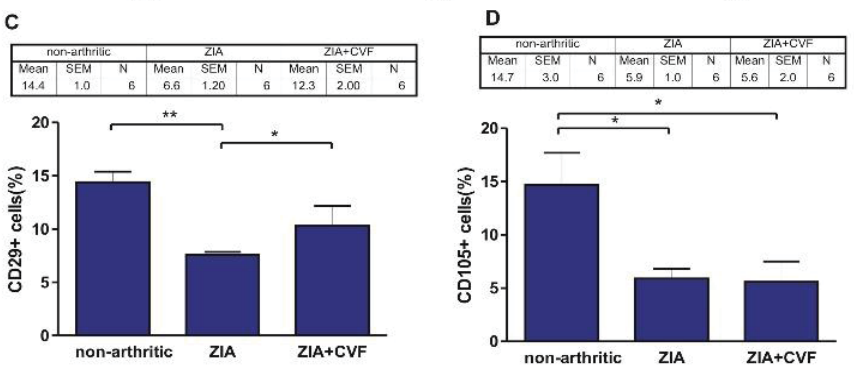

Figure 2: Influence of CVF-treatment on in vitro differentiation of fibroblast and mesenchymal cells. BM cells obtained from nonarthritic mice, mice with ZIA and pretreated with CVF mice with ZIA were collected at day 3 and cultured for 10 days with partial change of supplemented media. Representative histograms of mesenchymal CD29+ cells (A) and fibroblast-like CD105+ cells (B). Graphical presentation of CD29+cells (C) and CD105+ cells (D). Results are from two independent experiments (in each $n=10$ /per group) and expressed as means $\pm S E M,{ }^{*} P<0.05$, $\star \star x<0.01$ one way ANOVA. 


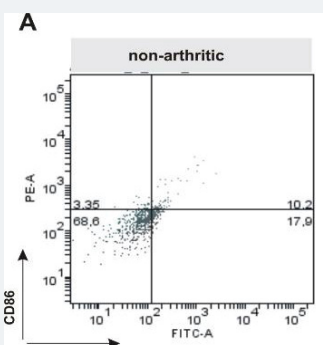

B

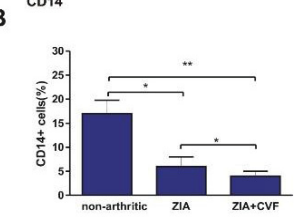

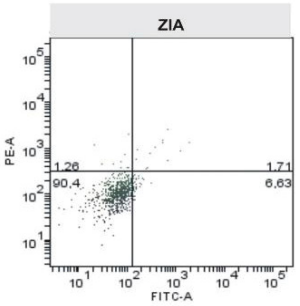

C

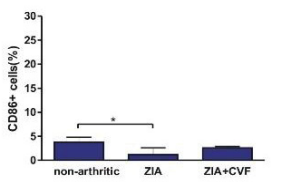

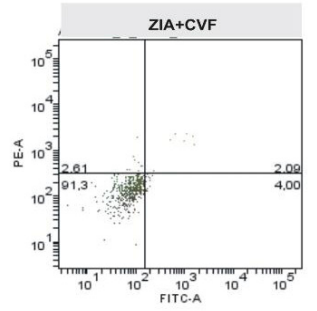

D

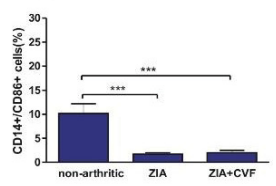

Figure 3: Effect of decomplementation on CD14 and CD86 expression. BM cells obtained from nonarthritic mice, mice with ZIA and pretreated with CVF mice with ZIA were collected at day 3 and the percentage of immature CD14+ dendritic cells and mature CD86+ dendritic cells (A) was determined and graphical presentation of data (B), (C) and (D). Results are from two independent experiments (in each $n=10 /$ per group) and expressed as means $\pm S E M$, ${ }^{\star} P<0.05,{ }^{\star *} P<0.01,{ }^{\star}{ }^{*} P<0.001$, one way ANOVA.

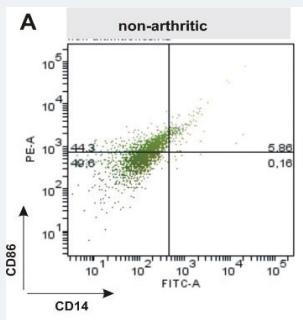

B

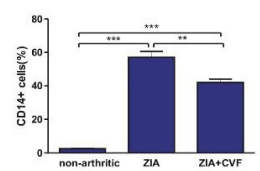

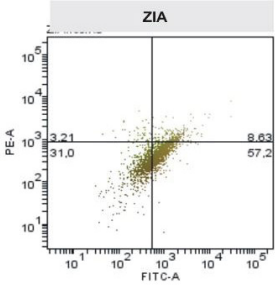

C

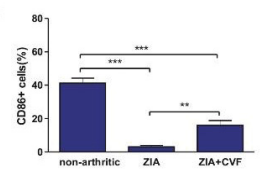

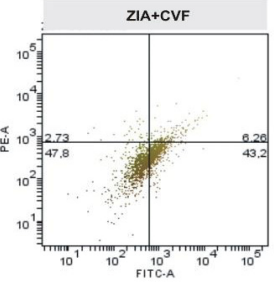

D

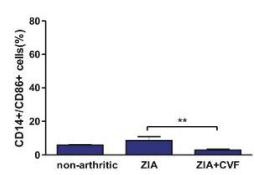

Figure 4: Influence of CVF-treatment on in vitro differentiation of dendritic cells. BM cells obtained from nonarthritic mice, mice with ZIA and pretreated with CVF mice with ZIA were collected at day 3 and stimulated for 6 days with GM-CSF+IL-4. Representative dot plots of CD14+ and CD86+ cells (A) and graphical presentation (B, C and D). Results are from two independent experiments (in each $n=10 /$ per group) and expressed as means $\pm S E M, * \star P<0.01$, $\star \star \star P<0.001$ one way ANOVA.

A
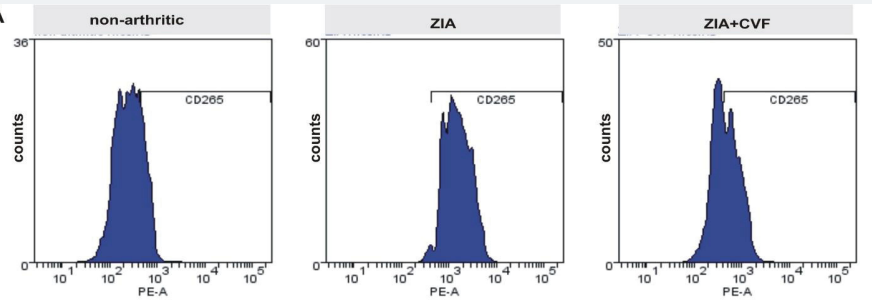

B
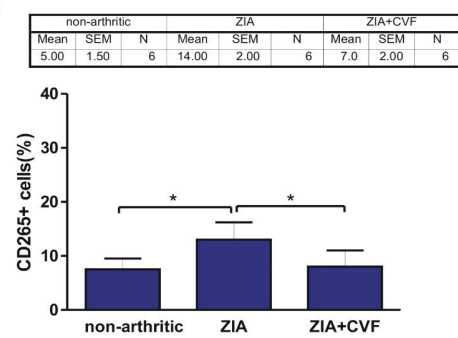

Figure 5: Effect of decomplementation on CD265 (RANK) expression. BM cells obtained from nonarthritic mice, mice with ZIA and pretreated with CVF mice with ZIA were collected at day 3 and the percentage of CD265+ cells (A) and graphical presentation (B). Results are from two independent experiments (in each $n=10 /$ per group) and expressed as means \pm SEM, $* P<0.05$, one way ANOVA. 

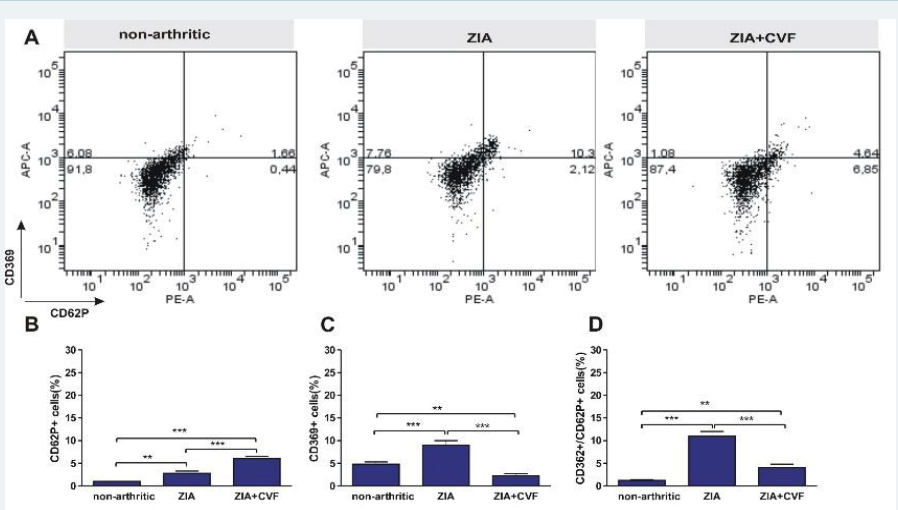

Figure 6: Influence of complement depletion on the differentiation of CD62P+ megakaryocyte and Dectin1 CD369+ cells. BM cells obtained from nonarthritic mice, mice with ZIA and pretreated with CVF mice with ZIA were collected at day 3 and the percentage of CD62P+ and mature CD369+ cells (A) was determined and graphical presentation of data (B), (C) and (D). Results are from two independent experiments (in each $n=10 /$ per group) and expressed as means \pm SEM, $\mathrm{n}=10,{ }^{\star *} P<0.01,{ }^{\star \star *} P<0.001$, one way ANOVA.

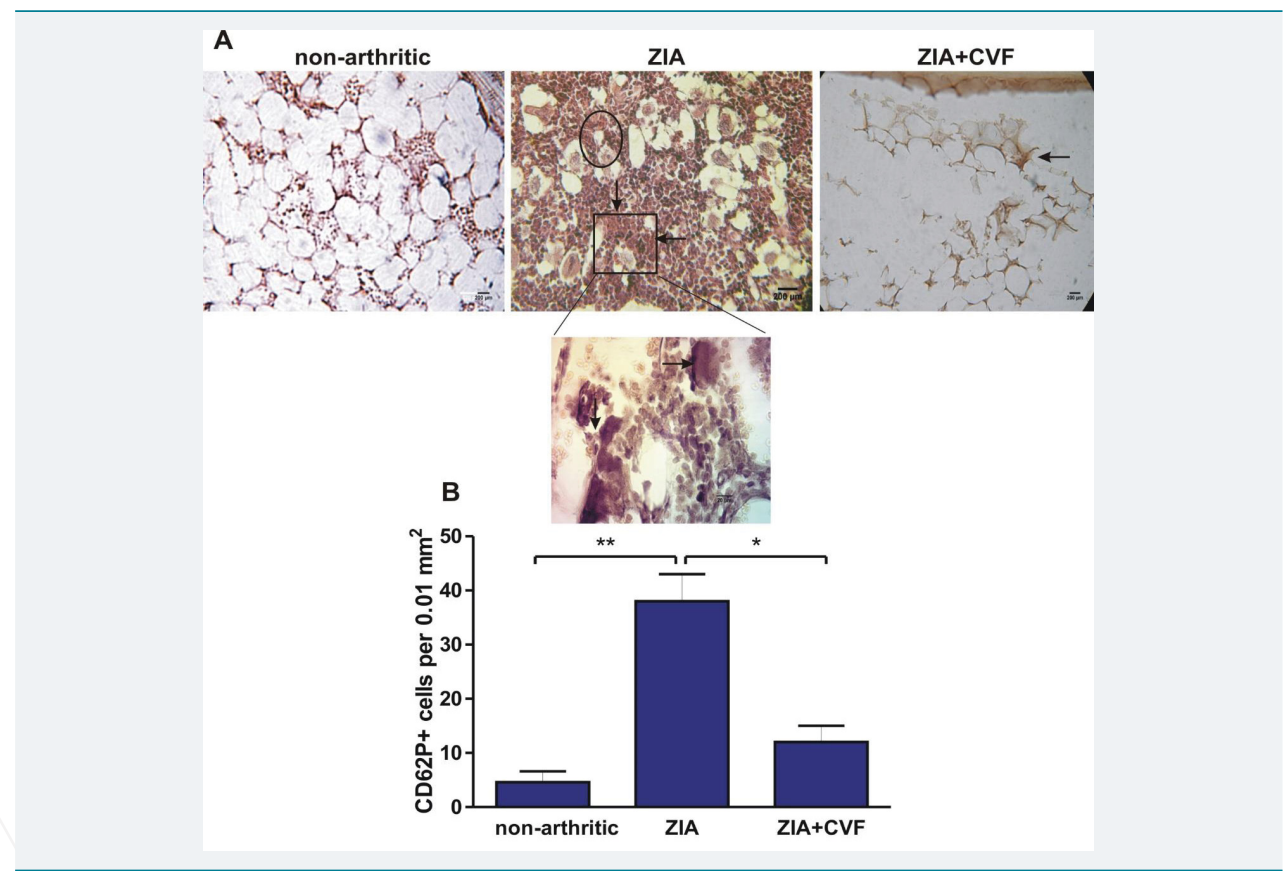

Figure 7: Immunochistochemically stained CD62P+ cells in BM. Sagital joint sections from nonarthritic mice, mice with ZIA and pretreated with CVF mice with ZIA at day 3 (A). Black arrows point on giant CD62P+ megakaryocytes, greater magnification below and groups of platelets in circle. Graphical presentation of data (B). Results are from three independent experiments (in each $n=5$ /per group)and expressed as means \pm SEM, $n=10,{ }^{*} P<0.05,{ }^{\star} * P<0.01$, one way ANOVA.

disease perpetuation. DCs cooperate with synoviocytes and macrophages and produce inflammatory mediators that drive RA pathology [30,31]. Under normal conditions, migration of resting DC precursors into tissues and into secondary lymphoid organs occurs permanently in the absence of inflammatory events. Preseent data showed that the development of ZIA is related to a decrease of immature and mature DCs in bone marrow and even greater decrease in concern to immature cells was observed in CVF-treated group. As a consequence less DCs will be released into the circulation and reside in tissues. In vitro cultivation of BM cells in the presence of GM-CSF and $\mathrm{IL}+4$ showed that cells obtained from arthritic mice were less susceptible to the stimulation hence the percentage of mature DCs was negligible compared to non-arthritic.

$\mathrm{T}$ cells require many receptor-ligand interactions to become activated and to carry out their tissue-destructive role in RA. Activated cells produce IL-17 that has independent and synergistic effects with other proinflammatory cytokines in the 
synovium to induce further cytokine release, matrix metalloproteinase production, and osteoclastogenesis. RANKL is a crucial regulator of osteoclastogenesis and bone erosion which binds to its unique receptor activator of NF-KB (RANK) [32,33]. IL-17 upregulates CD265 (RANK ligand) expression on chondrocytes and osteoblasts and acts on chondrocyte metabolism by reducing proteoglycan synthesis and enhancing cartilage degradation [34]. Our previous investigation demonstrated that BM cells obtained from ZIA mice injected with CVF showed inhibited OC formation induced by M-CSF+RANKL [24]. In this study we investigated in vivo the influence of low complement activity on the osteoclast generation in BM. CD265 positive cells were detected at the early phase of arthritis development to a great extent the percentage of these cells being lowered in decomplemented mice. Thus, destructive joint processes can be successfully limited through modulation of complement activity.

During complement activation C3 component is cleaved into C3a and des-ArgC3a that leads to modulation of MK unresponsiveness to stromal cell-derived factor 1 (SDF-1) and to regulation of platelets in inflammation [35]. MKs could influence bone formation and bone remodeling triggering osteoblast proliferation and/or inhibition of OC development. There is such possibility if viable MKs persist for a longer time in bone marrow [36]. In vitro, zymosan is used for the study of innate immune responses, because it induces inflammatory cytokine production and can activate complement in the absence of immunoglobulins [37]. Zymosan is also able to enhance maturation of DCs and increases their production of IL-2 [38]. Having in view these facts, we examined the possibility zymosan injection and persistence to provoke an appearance of MKs and cells detecting zymosan. Cells expressing CD62P were found in arthritic mice was elevated in decomplemented mice. As this marker is expressed on platelets too we cannot determine the percentage of megakaryocytes only. As well as it deserves further investigations on the role of complement activity in megakaryocytedependant processes in joint inflammation. Dectin1 positive cells were established in non-injected with zymosan mice, reasonably increased in arthritic mice. Also, Dectin1 positive megakaryocytes were observed in ZIA mice while CVF-treatment prevented the appearance of these pathology-related cells. Moreover, the presence of MKs presented at the site of inflammation was confirmed immunohistochemically. Chronic inflammation during erosive conditions such as rheumatoid arthritis leads to dysregulated differentiation and proliferation of bone cells, inflammation of synovial membrane and bone marrow, and degradation of cartilage and bone. CVF injection provokes C3 and C5 depletion for 5 days so the present results at day 3 of arthritis corresponded to the situation when complement cascade was blocked. Low complement activity altered mesenchymal and fibroblast-like cells and the ratio immature/mature DCs (Figure 8). Future experiments will help elucidation the role of complement in megakaryocyte differentiation and the consequences of inhibited platelet generation. These results can be used for determining the role of complement system at different stages of rheumatoid arthritis and can support finding of new therapeutic approaches.

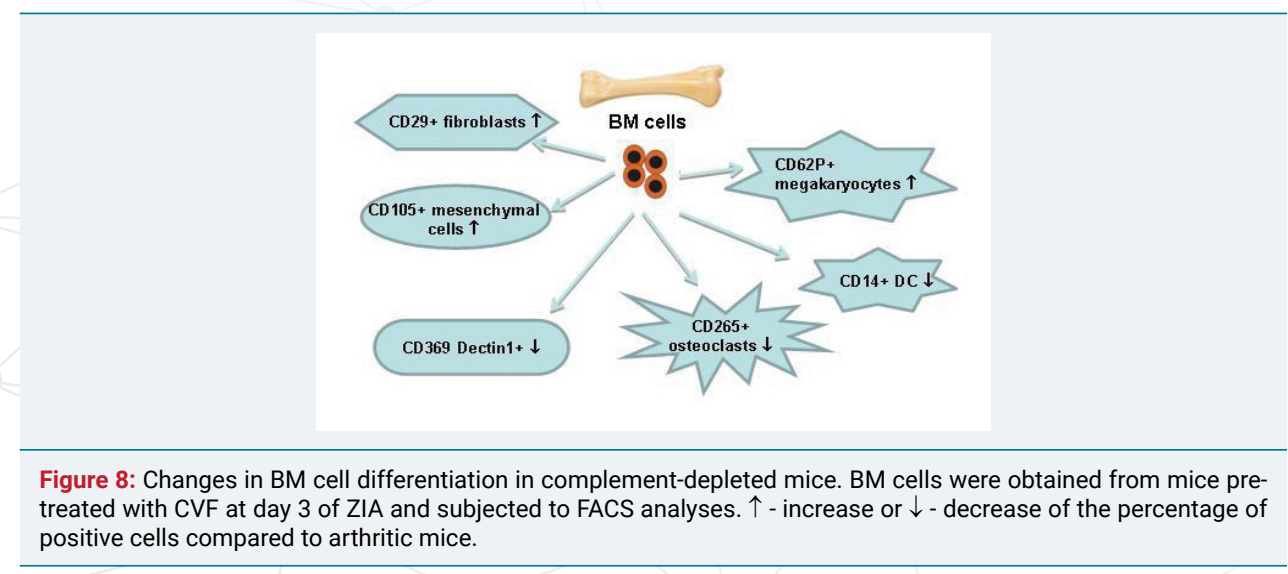




\section{Acknowledgement}

P. Ganova was supported by a Programme for young scientists and PhD students of the Bulgarian Academy of Sciences, grant DFNP-17-40/26.07.2017.

\section{References}

1. Kawai T, Matsuyama T, Hosokawa $Y$, Makihira S, Seki M, et al. B and T lymphocytes are the primary sources of RANKL in the bone resorptive lesion of periodontal disease. Am J Pathol. 2006; 169: 987998. Ref.: https://goo.gl/Axuf7P

2. Okroj $M$, Heinegård $D$, Holmdahl R, Blom AM. Rheumatoid arthritis and the complement system. Ann Med. 2007; 39: 517-530. Ref.: https://goo.gl/T4hFxH

3. Ricklin D, Hajishengallis G, Yang K, Lambris JD. Complement: a key system for immune surveillance and homeostasis. Nat Immunol. 2010; 11: 785-797. Ref.: https://goo.gl/L9XmUX

4. Konttinen YT, Ceponis A, Meri S, Vuorikoski A, Kortekangas $\mathrm{P}$, et al. Complement in acute and chronic arthritides: assessment of C3c, C9, and protectin (CD59) in synovial membrane. Ann Rheum Dis. 1996; 55: 888-894. Ref.: https://goo.gl/QcXZaA

5. Neumann E, Barnum SR, Tarner IH, Echols J, Fleck M, et al. Local production of complement proteins in rheumatoid arthritis synovium. Arthritis Rheum. 2002; 46: 934-945. Ref.: https://goo.gl/VzNUpi

6. O'Gradaigh D, Compston JE. T-cell involvement in osteoclast biology: implications for rheumatoid bone erosion. Rheumatology (Oxford). 2004; 43: 122-130. Ref.: https://goo.gl/pe25MS

7. Sato $\mathrm{T}$, Abe $\mathrm{E}$, Jin $\mathrm{CH}$, Hong $\mathrm{MH}$, Katagiri $\mathrm{T}$, et al. The biological roles of the third component of complement in osteoclast formation. Endocrinology. 1993; 133: 397-404. Ref.: https://goo.gl/adpH2m

8. Wang $X$, Wang Y, Gou W, Lu Q, Peng J, et al. Role of mesenchymal stem cells in bone regeneration and fracture repair: a review. Int Orthop. 2013; 37: 2491-2498. Ref.: https://goo.gl/sy5tJ5

9. Farini A, Sitzia C1, Erratico S1, Meregalli M1, Torrente Y. Clinical applications of mesenchymal stem cells in chronic diseases. Stem Cells Int. 2014; 2014: 306573. Ref.: https://goo.gl/uvRvMg

10. Kristjánsson $B$, Honsawek $S$. Mesenchymal stem cells for cartilage regeneration in osteoarthritis. World J Orthop. 2017; 8: 674-680. Ref.: https://goo.gl/RVv2Nz

11. Duff SE, Li C, Garland JM, Kumar S. CD105 is important for angiogenesis: evidence and potential applications, FASEB J. 2003; 17: 984-992. Ref.: https://goo.gl/UkUM2y

12. Lutzky V, Hannawi S, Thomas R. Cells of the synovium in rheumatoid arthritis. Dendritic cells. Arthritis Res Ther. 2007; 9: 219. Ref.: https://goo.gl/ung6ed

13. Sallusto F, Lanzavecchia A. Mobilizing dendritic cells for tolerance, priming, and chronic inflammation. J Exp Med. 1999; 189: 611-614. Ref.: https://goo.gl/7Sfk6N

14. Apostolopoulos V, Thalhammer T, Tzakos AG, Stojanovska L. Targeting antigens to dendritic cell receptors for vaccine development. J Drug Deliv. 2013; 2013: 869718 Ref.: https://goo.gl/B5kUTb

15. LeibundGut-Landmann S, Gross O, Robinson MJ, Osorio F, Slack EC, et al. Syk- and CARD9dependent coupling of innate immunity to the induction of $T$ helper cells that produce interleukin 17. Nat Immunol. 2007; 8: 630-638. Ref.: https://goo.gl/jnmsnS

16. Gerosa F, Baldani-Guerra B, Lyakh LA, Batoni G, Esin S, et al. Differential regulation of interleukin 12 and interleukin 23 production in human dendritic cells. J Exp Med. 2008; 205: 1447-1461. Ref.: https://goo.gl/JZgKad

17. Nakeff A, Maat B. Separation of megakaryocytes from mouse bone marrow by velocity sedimentation Blood. 1974; 43: 591-595. Ref.: https://goo.gl/8WZ9Qp

18. Zou Z, Schmaier AA, Cheng L, Mericko P, Dickeson SK, et al. Negative regulation of activated alpha-2 integrins during thrombopoiesis. Blood. 2009; 113: 6428-6439. Ref.: https://goo.gl/AQkrCF

19. Arbesu I, Bucsaiova M, Fischer MB, Mannhalter C. Platelet-borne complement proteins and their role in platelet-bacteria interactions. J Thromb Haemost. 2016; 14: 2241-2252. Ref.: https://goo.gl/BZyCPZ

20. Teitelbaum SL. Bone resorption by osteoclasts. Science. 2000; 289: 1504-1508. Ref.: https://goo.gl/o1FPZg

21. Boyce BF. Advances in the regulation of osteoclasts and osteoclast functions. J Dent Res. 2013; 92: 860-867. Ref.: https://goo.gl/rFcmvG 
22. Teitelbaum SL. The osteoclast and its unique cytoskeleton. Ann N Y Acad Sci. 2011; 1240: 14-17. Ref.: https://goo.gl/dDERHq

23. Belenska-Todorova L, Gyurkovska V, Ivanovska N. How complement activation influences the development of chronic synovitis in a mouse model of rheumatoid arthritis. Scand J Rheumatol. 2016; 45: 13-22. Ref.: https://goo.gl/tjJ7us

24. Ganova P, Gyurkovska V, Belenska-Todorova L, Ivanovska N. Functional complement activity is decisive for the development of chronic synovitis, osteophyte formation and processes of cell senescence in zymosan-induced arthritis. Immunol Lett. 2017; 190: 213-220. Ref.: https://goo.gl/6jwmK1

25. Buckley CD, Pilling D, Lord JM, Akbar AN, Scheel-Toellner D, et al. Fibroblasts regulate the switch from acute resolving to chronic persistent inflammation. Trends Immunol. 2001; 22: 199-204. Ref.: https://goo.gl/w2rV1j

26. Müller-Ladner U, Pap T, Gay RE, Neidhart M, Gay S. Mechanisms of disease: the molecular and cellular basis of joint destruction in rheumatoid arthritis. Nat Clin Pract Rheumatol. 2005; 1: 102-110. Ref.: https://goo.gl/Nv5BMj

27. Arend WP, Mehta G, Antonioli AH, Takahashi M, Takahashi K, et al. Roles of adipocytes and fibroblasts in activation of the alternative pathway of complement in inflammatory arthritis in mice. $\mathrm{J}$ Immunol. 2013; 190: 6423-6433. Ref.: https://goo.gl/7B9V1x

28. Guc D, Gulati P, Lemercier C, Lappin D, Birnie GD, et al. Expression of the components and regulatory proteins of the alternative complement pathway and the membrane attack complex in normal and diseased synovium. Rheumatol Int. 1993; 13: 139-146. Ref.: https://goo.gl/nwLmWj

29. Lefèvre S, Knedla A, Tennie C, Kampmann A, Wunrau C, et al. Synovial fibroblasts spread rheumatoid arthritis to unaffected joints. Nat Med. 2009; 15: 1414-1420. Ref.: https://goo.gl/ukVcP8

30. Leung BP, Conacher M, Hunter D, McInnes IB, Liew FY, et al. A novel dendritic cell-induced model of erosive inflammatory arthritis: distinct roles for dendritic cells in T cell activation and induction of local inflammation. J Immunol. 2002; 169: 7071-7077. Ref.: https://goo.gl/ukyXSt

31. Thomas R, Davis LS, Lipsky PE Rheumatoid synovium is enriched in mature antigen-presenting dendritic cells. J Immunol. 1994; 152: 2613-2623. Ref.: https://goo.gl/2E4Wfp

32. Dougall WC, Glaccum M, Charrier K, Rohrbach K, Brasel K, et al. RANK is essential for osteoclast and lymph node development. Genes Dev. 1999; 13: 2412-2424. Ref.: https://goo.gl/M5AoWD

33. Kong YY, Yoshida H, Sarosi I, Tan HL, Timms E, et al. OPGL is a key regulator of osteoclastogenesis, lymphocyte development and lymph-node organogenesis. Nature. 1999; 397: 315-323. Ref.: https://goo.gl/VgBFef

34. Lubberts $\mathrm{E}$, Koenders $\mathrm{MI}$, van den Berg WB The role of T-cell interleukin-17 in conducting destructive arthritis: lessons from animal models. Arthritis Res Ther. 2005; 7: 29-37. Ref.: https://goo.gl/EXBJVk

35. Wysoczynski M, Kucia M, Ratajczak J, Ratajczak MZ. Cleavage fragments of the third complement component (C3) enhance stromal derived factor-1 (SDF-1)-mediated platelet production during reactive post bleeding thrombocytosis. Leukemia. 2007; 21: 973-982. Ref.: https://goo.gl/U1ZLia

36. Ciovacco WA, Cheng YH, Horowitz MC, Kacena MA. Immature and mature megakaryocytes enhance osteoblast proliferation and inhibit osteoclast formation. J Cell Biochem. 2010; 109: 774-781. Ref.: https://goo.gl/j58HZn

37. Underhill DM. Macrophage recognition of zymosan particles. J Endotoxin Res. 2003; 9: 176-180. Ref.: https://goo.gl/Qof2oK

38. Granucci F, Feau S, Angeli V, Trottein F, Ricciardi-Castagnoli P. Early IL-2 production by mouse dendritic cells is the result of microbial-induced priming. J Immunol. 2003; 170: 5075-5081. Ref.: https://goo.gl/CRn9Es 